\title{
ASSESSMENT OF POST-OPERATIVE VELOPHARYNGEAL CLOSURE IN CLEFT PALATE PATIENTS USING CONE-BEAM COMPUTED TOMOGRAPHY: A PILOT STUDY
}

\author{
Artúr Kesztyüs', Laura Pálvölgyi', Reinhilde Jacobs²,3, Krisztian Nagy' \\ ${ }^{1}$ The Cleft Centre, ${ }^{\text {st }}$ Department of Paediatrics, Semmelweis University Budapest, Hungary \\ 20MFS IMPATH Research Group, Department of Imaging and Pathology, Faculty of Medicine, University of Leuven, Belgium \\ ${ }^{3}$ Department of Dental Medicine, Karolinska Institute, Sweden
}

\begin{abstract}
INTRODUCTION: Cleft lip and palate can induce velopharyngeal insufficiency (VPI) that can be detected with numerous imaging techniques. For specific treatment, however, it is essential to implement the appropriate imaging method.

ОвјестіVEs: To introduce a novel technology related to cone-beam computed tomography (CBCT) for assessing movements of the soft palate as a potential diagnostic tool for VPI.

MATERIAL AND METHODS: Nine participants with and without VPI underwent CBCT examination. Upper airway two-dimensional video images (CineX view) enabled functional assessment. To achieve standardization, previously defined CBCT image quality parameters have been applied. Soft tissue contour, movement, and contact between these structures were assessed by two observers.

RESULTS: Soft palate contour and movements were visualized sufficiently to assess presence of velopharyngeal closure in each case. These images enabled qualitative and dynamic assessment of velopharyngeal closure, correlating with VPI.

ConcLusions: $\mathrm{CBCT}$ is feasible to detect soft palate movement and velopharyngeal closure as a rapid, non-invasive procedure.
\end{abstract}

KEY wORDS: cleft palate, CBCT, velopharyngeal, dynamic.

J Stoma 2021; 74, 2: 65-69

DOI: https://doi.org/10.5114/jos.2021.106501

\section{INTRODUCTION}

Velopharyngeal insufficiency (VPI) is a speech difficulty that occurs when the soft palate is unable to close tightly against the back of the throat, resulting inadequate closure of the nasal airway during speech. VPI can be observed in cleft lip and palate (CLP) patients due to the anatomic irregularity of the soft palate. Hypernasality, decreased vocal intensity, nasal turbu- lence, or emission represent the major signs, which indicate insufficient velopharyngeal function (VPI) [1]. Several treatment options are available for this disorder, with autologous fat grafting as one of the least invasive techniques [2].

Numerous visualization methods exist to assess VPF, such as video fluoroscopy, flexible nasopharyngoscopy, helical computer tomography, and magnetic resonance imaging. Possible risks and benefits of each method
JOURNAL OF STOMATOLOGY CZASOPISMO STOMATOLOGICZNE
AdDRESS FOR CORRESPONDENCE: Krisztián Nagy, MD, PhD,

$1^{\text {st }}$ Department of Paediatrics, Bókay J. u. 53-54, 1083 Budapest, Hungary, phone: +3613343743, e-mail: nagykrisztian@me.com

ReCeIved: 10.02.2021 • ACCePTED: 25.03.2021 • Published: 04.06.2021 
should be considered carefully in order to obtain the appropriate individual treatment, since the ideal imaging method should be non-invasive, reproducible, and repeatable, with minimal risk to the child [3].

In the last few years, cone-beam computed tomography $(\mathrm{CBCT})$ has become more prevalent in maxillofacial imaging. CBCT is suitable for objective assessment of head and neck pathologies, including skeletal, dental, and upper airway deformities. Even if CBCT imaging has a major advantage in visualization of skeletal and dental structures, it has been clearly shown that upper airway structures and their borders can be adequately distinguished for volumetric analysis [4].

\section{OBJECTIVES}

The overall aim of this study was to evaluate if soft palate movement could be adequately visualized using CBCT, with an objective to validate its applicability as a clinical assessment tool for surgical management of VPI.

\section{MATERIAL AND METHODS}

The study included 9 children, aged between six and 10 years. We have obtained ethical approval from the Semmelweis University (Semmelweis University Ethical Committee approval No. 265/2019). The sample consisted of 9 participants who underwent CBCT examination, including 3 surgically managed unilateral or bilateral cleft lip and palate (UCLP or BCLP) patients with VPI, 3 surgically managed unilateral cleft lip and palate or cleft palate $(\mathrm{CP})$ patients without VPI, and 3 non-cleft patients as a control group (Table 1).

CBCT examinations were justified for indications of pre-operative planning of alveolar grafting combined with orthodontic assessment of the cleft patients. For the control group patients, three-dimensional images were taken to assess other dental deformities (impacted canine, mesiodens, etc.).

Images were acquired using the dynamic acquisition function of the CBCT device (CineX movie setting, NewTom VGi Evo, QR Verona, Cefla, Imola, Italy, operating at $110 \mathrm{kV}$ ), resulting a sequence of two-dimensional images to be converted and played as video. During the scans, the participants were asked to say the word 'coo-coo-reeh-coo', which is commonly used in speech therapy at our cleft center for assessing velopharyngeal function.

Age-specific radiation dose was estimated considering the field of view (FOV), beam qualities, and scan geometry settings using the Monte Carlo simulation framework. It included three parts: source modelling, angular projection modelling, and dose tracking. Data were analyzed to investigate the correlation of organ dose, effective dose (ED), and life attributable risk (LAR) patterns with age [5]. Subjective image quality was assessed by two observers (AK and $\mathrm{KN}$ ). In order to achieve standardization, an agreement on the diagnostic evaluation criteria was established between the observers. Subsequently, the following structures were examined for possible visualization and identification:

- soft tissue outlines and contour of the posterior pharyngeal wall,

- soft tissue outlines and contour of the soft palate,

- contact between the above-mentioned structures.

\section{RESULTS}

All subjects received a patient-specific radiation dose, ranging from 279 to 515 DAP (dose area product, $\mathrm{mGy} \times \mathrm{cm}^{2}$ ), depending on the orofacial morphology of individual patient. Radiation time varied between 0.78-1.5 seconds, using a default setting of 20 frames per second. The $\mathrm{mA}$ (milliampere) value remained $3 \mathrm{~mA}$, as it was a not variable parameter of the device.

TABLE 1. Patients' characteristics according to the indication of cone-beam computed tomography (CBCT) justification and the presence of velopharyngeal dysfunction

\begin{tabular}{|c|c|c|c|c|c|}
\hline Patient & Age of patient (years) & $\begin{array}{l}\text { Indication for CBCT } \\
\text { sjustification }\end{array}$ & $\begin{array}{l}\text { Post-op time after } \\
\text { palatoplasty (years) }\end{array}$ & Type of patient & Presence of VPI \\
\hline 1 & 10 & Impaction tooth, 23 & - & Non-cleft & No VPI \\
\hline 2 & 9 & Dilate ration tooth, 11 & - & Non-cleft & No VPI \\
\hline 3 & 10 & Impaction teeth, 11-12-13 & - & Non-cleft & No VPI \\
\hline 4 & 8 & $\mathrm{BCLP}$ & 7 & Cleft & No VPI \\
\hline 5 & 6 & UCLP, left & 5 & Cleft & No VPI \\
\hline 6 & 6 & UCLP, left & 5 & Cleft & No VPI \\
\hline 7 & 7 & $C P$ & 6 & Cleft & VPI \\
\hline 8 & 7 & $C P$ & 6 & Cleft & VPI \\
\hline 9 & 7 & UCLP, right & 6 & Cleft & VPI \\
\hline
\end{tabular}


The CBCT images were evaluated by two observers, and the following findings were described identically and independently by them:

- the movement of soft palate was clearly visible, allowing diagnostic findings regarding VPI; with the CineX movie setting (NewTom VGi Evo, QR Verona, Cefla, Imola, Italy), not only structures but also movements of the soft palate and posterior pharyngeal wall could be evaluated;

- the dorso-cranial elevation of the soft palate and ventro-caudal descent of the posterior pharyngeal wall were clearly distinguishable (Figure 1);

- in accordance with the prior diagnoses in the cases of both non-cleft and cleft lip and palate patients with-

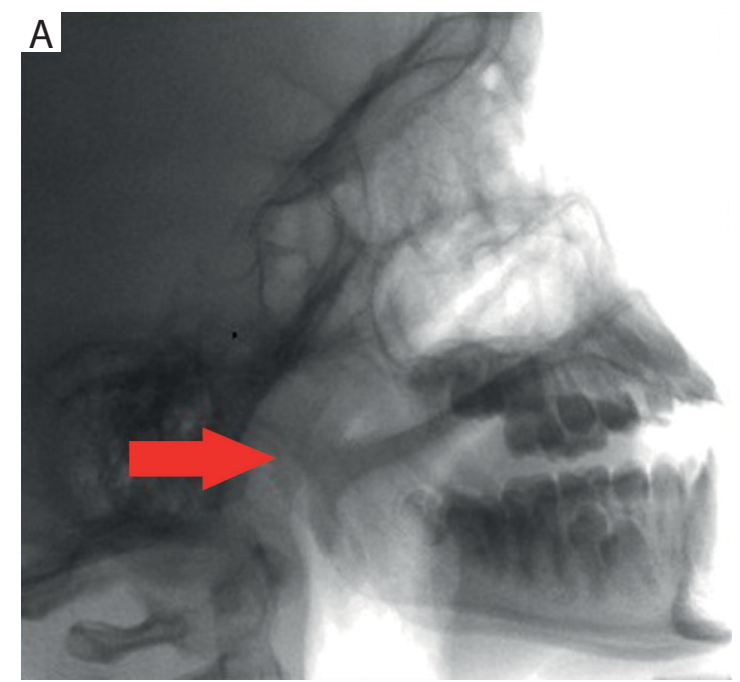

out VPI, proper contact between the soft palate and posterior velopharyngeal wall could be observed, resulting a sufficient closure (Figures 1 and 2);

- CBCT images of the cleft patients with VPI showed incomplete closure due to a shorter soft palate and poor levator palati muscle control (Figure 3).

\section{DISCUSSION}

For correction of VPI various surgical and non-surgical procedures were introduced. A multidisciplinary team is indispensable for effective diagnosis and treatment. Moreover, adequate visualization of VPF is mandatory

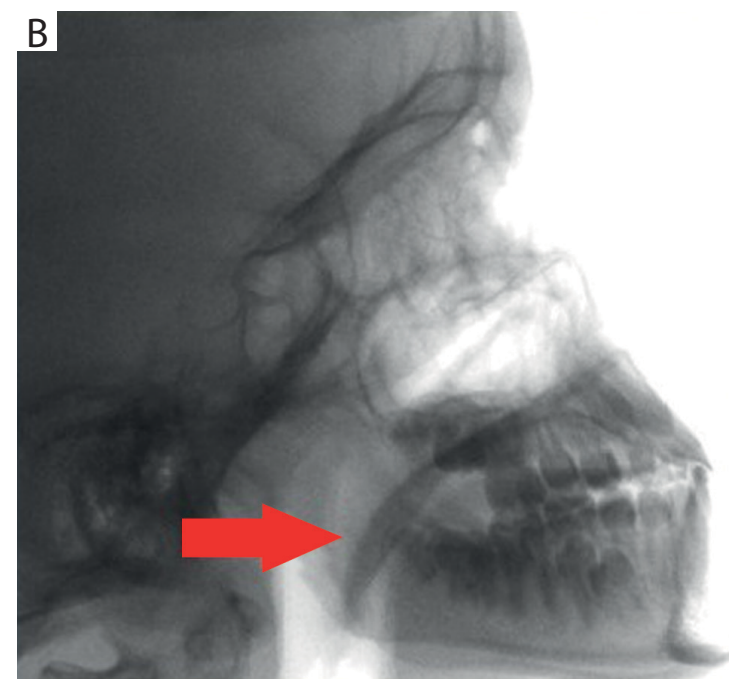

FIGURE 1. Patient 1 (non-cleft patient, no velopharyngeal insufficiency): maximum elevation of the soft palate (Figure $1 \mathrm{~A}$ ) and relaxed position of the soft palate (Figure 1 B). The red arrow indicates the position of the soft palate and sufficient velopharyngeal closure in Figure $1 \mathrm{~A}$
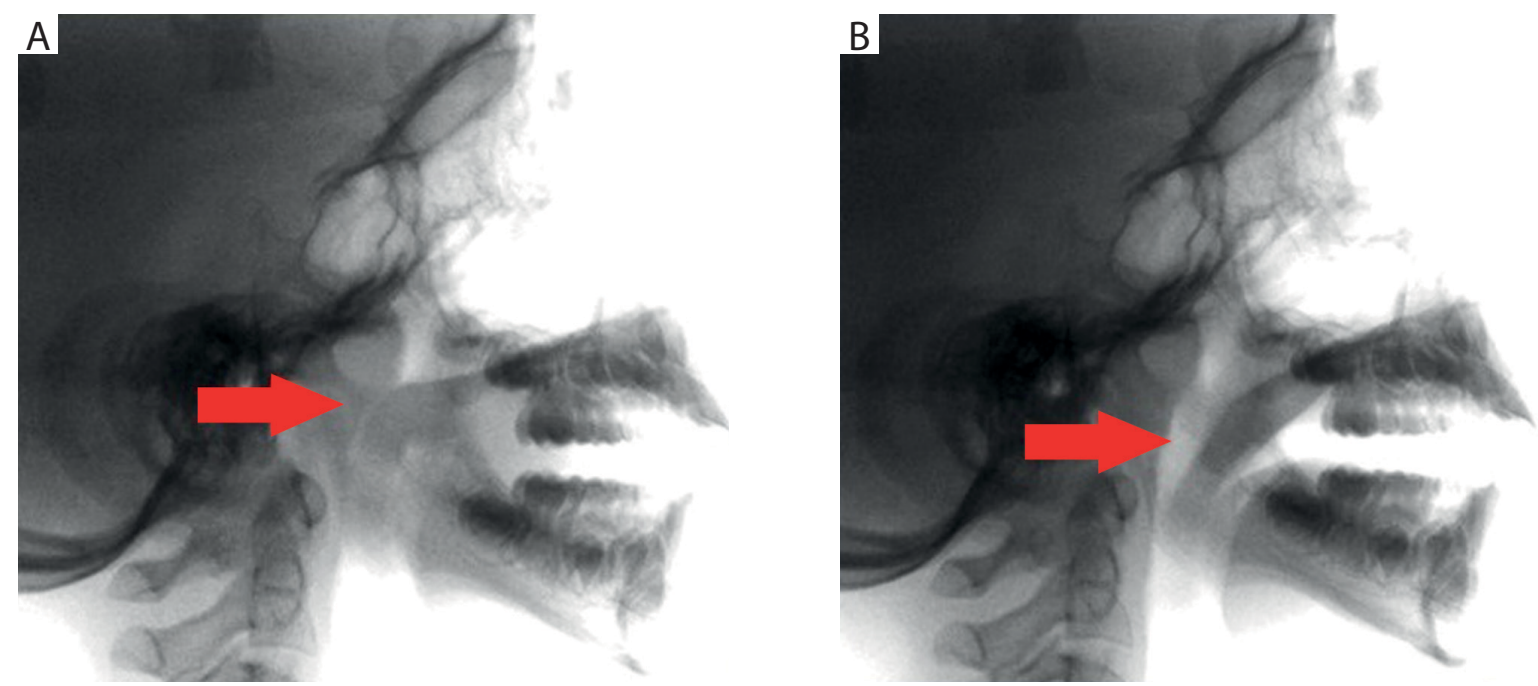

FIGURE 2. Patient 2 (post-palatoplasty, cleft patient, no velopharyngeal insufficiency): maximum elevation of the soft palate (Figure $2 \mathrm{~A}$ ) and relaxed position of the soft palate (Figure 2 B). The red arrow indicates the position of the soft palate and sufficient velopharyngeal closure in Figure $2 \mathrm{~A}$ 

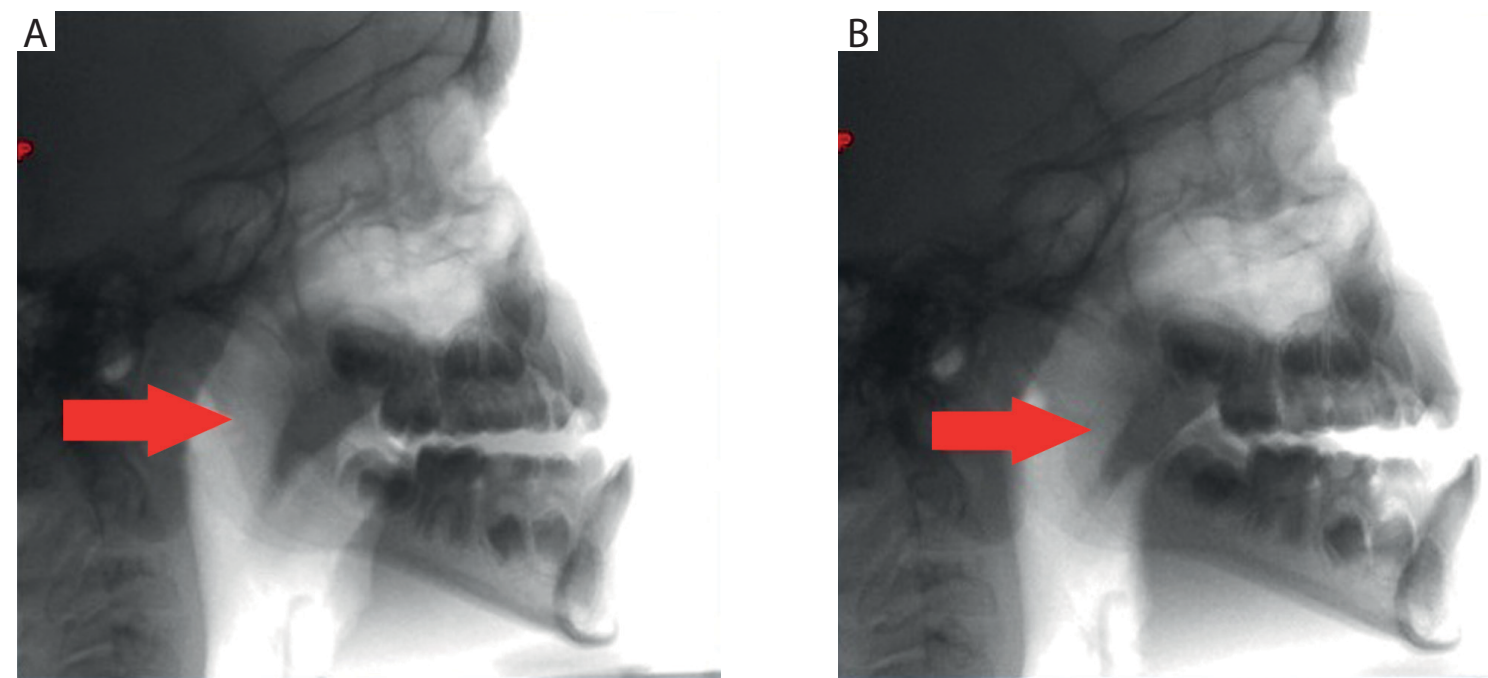

FIGURE 3. Patient 3 (post-palatoplasty, cleft patient, velopharyngeal insufficiency): maximum elevation of the soft palate (Figure $3 \mathrm{~A}$ ) and relaxed position of the soft palate (Figure $3 \mathrm{~B}$ ). The red arrow indicates the position of the soft palate and insufficient velopharyngeal closure in Figure $3 \mathrm{~A}$

for the decision-making in the treatment protocol. Several diagnostic methods may allow direct observation of the velopharyngeal closure or can even enable the visualization of velopharyngeal muscles.

Flexible nasopharyngoscopy is the most frequently used tool. It allows monitoring the anatomy and muscular function of the velopharynx during speech. The major limitation of this method is its invasive technique, which is hardly tolerated by younger patients [6].

Videofluoroscopy (VFS) and multiview fluoroscopy are both non-invasive diagnostic tools, and are among the commonly used radiographic diagnostic methods of VPF [5]. They allow measuring the structure and movement of the soft palate, including the extent and timing of closure. The drawback of this method is the multiple image acquisition, being time-consuming and inconvenient. Furthermore, VFS increases radiation dose to young children with CLP who are sensitive to repeated radiographic examinations [7].

Dynamic magnetic resonance imaging (MRI) could eliminate these limitations of the above-mentioned techniques, as it is a non-invasive and non-ionizing imaging technology. It enables the evaluation of the velopharyngeal muscle function and morphology, which is fundamental to assess the closure. MRI has been proven suitable to estimate the effectiveness of autologous fat transplantation as a therapy of mild VPI [8]. On the other hand, this imaging technique is incapable of detection of lateral velopharyngeal wall's motion, and determination of the closure pattern is less reliable. However, frequently, the use of MRI for such diagnosis is often hampered by high costs involved [9].

Conventional helical CT was the first imaging technology to detect complex craniofacial deformities and pathologies, with enhanced resolution. Compared to CBCT imaging technology, major limiting factors of using helical CT are high cost, its size, and elevated radiation dose [10].

CBCT also gained popularity in the non-invasive diagnostic imaging of dentofacial deformities (e.g., cleft palate patients), due to its ability to visualize threedimensional structures. Compared to traditional helical CT, CBCT results in a more detailed imaging of the bone, using lower radiation exposure, and leaving a smaller footprint, while being less expensive. It also allows in-office scanning, which is more convenient and comfortable for children. CBCT allows the 3D reconstruction of different anatomical structures in a high spatial resolution [11]. Additionally, CBCT-based data can be used for virtual models in biomechanical simulations. These models possess more considerable diagnostic value than other digital images [12].

Radiation dose for routine maxillofacial CBCT examination remains a pressing issue, where age and surrounding tissues are the key problems when determining radiation dose. Indeed, young individuals with developing tissues have a higher risk of cellular damage [13]. Therefore, pediatric head and neck image optimization has the highest priority to ensure the lowest achievable risk in young patients. Following principles, such as ALARA (as low as reasonably achievable) and ALADA (as low as diagnostically acceptable), CBCT imaging should not be used in routine orthodontic evaluation [14], but it is justified in the diagnostic imaging of complex malformations, including cleft lip and palate [15]. The prime challenge of imaging is to choose the optimal acquisition settings in order to limit radiation dose burden in pediatric patients. It has been suggested that the introduction of optimized imaging protocol is required for orofacial patients, and a sequence of diagnostic images has been proposed as an international recommendation 
based on European guidelines to achieve justification and optimization of imaging techniques [16]. Use of low exposure settings and narrow collimation progressively allowed for reduction of radiation dose and prevention of secondary malignancies [17]. Although some studies showed that reduction in tube current $(\mathrm{mA})$ resulted in acceptable image quality, lowering of the exposure level is limited [12].

In CBCT devices, such as NewTom VGi Evo (QR Verona, Cefla, Imola, Italy), default settings change patient-specifically, with the advantage to lower the dose and tube current modulation in smaller patients, thus meeting the above-mentioned requirements [5].

Our findings showed that the CineX movie setting (NewTom VGi Evo, QR Verona, Cefla, Imola, Italy) could be used as an effective tool to visualize the movements of the soft palate, and to assess the presence or absence of velopharyngeal closure; therefore, recognize the velopharyngeal insufficiency and evaluate the effect of various treatment options (e.g., autologous fat grafting).

\section{CONCLUSIONS}

CBCT imaging can be used as a diagnostic tool to visualize soft palate movements and to detect velopharyngeal closure. The amount of soft palate elevation can be assessed pre- and post-operatively, and as a rapid and non-invasive procedure, it can support surgeons and speech therapists in the diagnostic and therapeutic decision-making process.

\section{ETHICAL CONSIDERATIONS}

The research plan was compiled in accordance with the legislation in force and the Declaration of Helsinki of the World Medical Association (reference No: 23/ 2002.V.9).

\section{CONFLICT OF INTEREST}

The authors declare no potential conflicts of interest with respect to the research, authorship, and/or publication of this article.

\section{References}

1. Lewis JR, Andreassen ML, Leeper HA, Macrae DL, Thomas J Vocal characteristics of children with cleft lip/palate and associated velopharyngeal incompetence. J Otolaryngol 1993; 22: 113-117.

2. Bishop A, Hong P, Bezuhly M. Autologous fat grafting for the treatment of velopharyngeal insufficiency: state of the art. J Plast Reconstr Aesthet Surg 2014; 67: 1-8.

3. Rudnick EF, Sie KC. Velopharyngeal insufficiency: current concepts in diagnosis and management. Curr Opin Otolaryngol Head Neck Surg 2008; 16: 530-535.

4. Khare P, Reddy R, Gupta A, Sharva V, Gupta M, Singh P. Morphometric assessment of soft palate in oral submucous fibrosis using cone beam computed tomography: a cross sectional study. J Indian Acad Oral Med Radiol 2019; 31: 203-209.

5. Stratis A, Zhang G, Jacobs R, Bogaerts R, Bosmans H. The growing concern of the radiation dose in paediatric dental and maxillofacial CBCT: an easy guide for daily practice. Eur Radiol 2019; 29: 7009-7018.

6. Glade RS, Deal R. Diagnosis and management of velopharyngeal dysfunction. Oral Maxillofac Surg Clin North Am 2016; 28: 181-188.

7. Jacobs R, Pauwels R, Scarfe W, et al. Paediatric cleft palate patients show a 3 - to 5 -fold increase in cumulative radiation exposure from dental radiology compared with an age- and gender-matched population: a retrospective cohort study. Clin Oral Investig 2017; 22: 1783-1793.

8. Impieri D, Tønseth KA, Hide $\varnothing$, Feragen KJB, Høgevold HE, Filip C. Autologous fat transplantation to the velopharynx for treating mild velopharyngeal insufficiency: a 10-year experience. J Plast Reconstr Aesthet Surg 2019; 72: 1403-1410.

9. Fu M, Zhao B, Carignan C, et al. High-resolution dynamic speech imaging with joint low-rank and sparsity constraints. Magn Reson Med 2015; 73: 1820-1832.

10. Perry JL, Mason K, Sutton BP, Kuehn DP. Can dynamic MRI be used to accurately identify velopharyngeal closure patterns? Cleft Palate Craniofac J 2018; 55: 499-507.

11. Nakajima A, Sameshima GT, Arai Y, Homme Y, Shimizu N, Dougherty Sr H. Two- and three-dimensional orthodontic imaging using limited cone beam-computed tomography. Angle Orthod 2005; 75: 895-903.

12. Periago DR, Scarfe WC, Moshiri M, Scheetz JP, Silveira AM, Farman AG. Linear accuracy and reliability of cone beam CT derived 3-dimensional images constructed using an orthodontic volumetric rendering program. Angle Orthod 2008; 78: 387-395.

13. Pauwels R, Seynaeve L, Henriques JC, et al. Optimization of dental CBCT exposures through mAs reduction. Dentomaxillofac Radiol 2015; 44: 2015-2108.

14. Oenning AC, Jacobs R, Pauwels R, Stratis A, Hedesiu M, Salmon B. Cone-beam CT in paediatric dentistry: DIMITRA project position statement. Paediatr Radiol 2018; 48: 308-316.

15. Silva MA, Wolf U, Heinicke F, Bumann A, Visser H, Hirsch E Cone-beam computed tomography for routine orthodontic treatment planning: a radiation dose evaluation. Am J Orthod Dentofacial Orthop 2008; 133: 640.e1-640.e5.

16. De Mulder D, Cadenas de Llano-Pérula M, Willems G, Jacobs R, Dormaar JT, Verdonck A. An optimized imaging protocol for orofacial cleft patients. Clin Exp Dental Res 2018; 4: 152-157.

17. Gijbels F, Sanderink G, Wyatt J, Van Dam J, Nowak B, Jacobs R. Radiation doses of indirect and direct digital cephalometric radiography. Br Dental J 2004; 197: 149-152. 